\title{
Advances in systemic lupus erythematosus
}

\author{
Su-Ann Yeoh, Sofia Sapeta Dias, David A. Isenberg
}

Su-Ann Yeoh is a Specialty Registrar in Rheumatology, University College Hospital London. Competing interests: none declared. Contact email: su-ann.yeoh@nhs.net

Sofia Sapeta Dias MD is a Specialty Registrar in Internal Medicine, Internal Medicine Department I, Hospital de Santa Maria, Lisbon, Portugal. Competing interests: none declared.

David A Isenberg MD FRCP FAMS is Academic Director of Rheumatology at University College London, London, UK. Competing interests: none declared. Contact email: d.isenberg@ucl.ac.uk

\section{Abstract}

Systemic lupus erythematosus (SLE) is a chronic autoimmune disease with a heterogeneous course and systemic involvement. It is the result of a complex pathogenic pathway that culminates in autoantibody formation. The interaction between environmental triggers and genetic susceptibility is key in this process. Genome-Wide Association Study (GWAS) technology has allowed the recognition of over 80 loci associated with SLE that lead to the formation of key proteins, each of which contributes a small increase to the risk. Advances in the management of the disease include new validated standardized tools to capture disease activity, damage and quality of life, for clinical and research purposes. The prognosis of SLE has much improved in the last 50 years due to better general management and specific treatment, including better use of immunosuppressives and development of a new group of drugs - biologic therapy.

\section{Keywords}

Disease activity index; management; pathogenesis; systemic lupus erythematosus; treatment

Word count 6482

Number of figures 0

Number of tables 3

\section{Introduction}

Systemic lupus erythematosus (SLE) is a chronic multi-systemic autoimmune disease with a very heterogeneous pattern of clinical and serological manifestations. Its course differs in different individuals and is unpredictable within the same patient over time, which makes it very interesting as well as challenging to manage.

The pathogenesis of SLE is the result of interactions between genes, hormones and the environment but its precise development is mostly unknown. Recently, over 80 risk genes for the disease have been described. Certain genetic features are also associated with increased disease activity.

In the last 30 years, major efforts have been made to define some key aspects of the condition, notably disease activity and damage, using standardized indices. These tools are essential for the 
comparison of different cohorts, assessment of disease progression and prognosis, and measurement of response to treatment. This approach is particularly important now that new biologic drugs are beginning some encouraging signs of being effective in lupus.

This review focuses mostly on the recent advances in understanding and managing SLE.

\section{Understanding}

\section{Epidemiology}

SLE is a rare disease. Estimates of its incidence are in the range 1-10 per 100,000 person-years and of prevalence from 20 to over 200 per 100,000.1 The incidence may be increasing, probably due to higher awareness of the disease The prevalence has also been thought to be increasing, which may be reflective of an improvement in survival rates as well as its chronicity. SLE is more frequent, more severe in African, Hispanic, Chinese and Asian descendants. These patients have more haematological, serosal, neurologic and renal manifestations in general, although clinical profiles vary in specific populations. The study of the LUMINA (Lupus in Minorities: Nature vs Nurture) cohort concluded that, especially in AfricanAmerican and Hispanic populations (from Texas), there is an association with high disease activity and damage. Besides ethnicity, other predictors of damage are age, disease duration, disease activity and corticosteroids.

Socioeconomic status is also associated with a worse prognosis, $\underline{2}$ particularly with respect to the later manifestations of the disease. Poor social support is more commonly found among ethnic minorities, which makes it difficult to distinguish their independent contributions.

SLE is more frequent among women of child-bearing age in a ratio that varies between populations but is on average 10:1. Although the age of diagnosis also depends on ethnicity, it is most commonly described to be in the 3rd and 4th decades of life. Males and females show little difference in their disease manifestations or severity, though presentation at the extremes of life is associated with increased severity. 1

\section{Pathogenesis}

There is a complex interaction between gene susceptibility, hormonal influences and environmental triggers with a breakdown of immune tolerance, resulting in autoantibody production and the consequent dysregulation of the inflammatory response leading to the induction and maintenance of the disease.

\section{Genetic factors}

The genetic component in SLE pathogenesis was first suggested by the evident concordance between monozygotic twins in $24-69 \%$ compared to $1-5 \%$ in dizygotic twins, $\underline{4}$ and $\underline{5}$ and also by the different prevalence in various ethnic groups. An 8-20-fold increased risk of developing SLE has been reported in siblings of SLE patients. Some rare genetic mutations associated with familial SLE-like disease, transmitted in a Mendelian fashion, have been identified but these account for only $1-2 \%$ of cases. The 
discovery of these rare genetic mutations also helped understanding of some aspects of pathogenesis of this disease, since these mutations interfere with the function of the classical complement pathway (C1q, C2 and C4) and Fc gamma receptors, which are both implicated in disease pathogenesis.

In the last decade, with the development of Genome-Wide Association Study (GWAS) technology, over 80 loci with common variants have been shown to have a confirmed association with SLE. These genes lead to the formation of key proteins involved in innate and adaptive immunity. $\underline{5}$ Each appears to make a small contribution to the complex pathogenesis of lupus, which suggests that they work in a cumulative way.

One of the chromosomal regions having the strongest association with SLE is the human leucocyte antigen (HLA) locus, especially the class II region containing HLA-DRB1, DQA1 and DQB1. There are also associations of some of these loci with specific clinical features (e.g. DRB1 and renal disease) $)^{5}$ and serologic features (e.g. DR2 and anti-Sm antibodies, and DR3 and anti-Ro antibodies). $\underline{6}$

Though hormonal influences have a greater importance in determining the higher prevalence of SLE in women, some X-chromosome linked genes have been described that might contribute to this (e.g. IRAK1, its neighbour gene MECP2 and presence of CD40L). $\frac{5}{}$

In the last decade, the importance of the interferon (IFN) signature in the pathogenesis of SLE has been recognized. IFNa is a key mediator in the activation of innate response and also in the adaptive immune system (normally in response to a viral infection). It enhances natural killer-cell activity, stimulates the maturation of antigen-presenting cells, prevents apoptosis of $\mathrm{T}$ cells, suppresses regulatory T cells, and promotes B cell differentiation and antibody production. In patients with SLE, IFNa expression is increased in the absence of appropriate stimuli, because of the over-expression of regulating genes, and IFNa concentration is associated with disease activity.

Besides the growing knowledge of the genetics of SLE, post-translational modifications are likely to be contributors to the complex inheritance and incomplete concordance between homozygotic twins. In SLE, epigenetic modifications such as abnormalities in DNA methylation and histones have been reported. 5 For example, an elevated IL- 6 concentration may contribute to proliferation of $B$ cells via DNA methylation.

\section{Environmental influences and triggers}

The importance of the environment has been suggested by epidemiologic studies. The 'prevalence gradient hypothesis' describes a higher prevalence of SLE in the Afro-Caribbean population living in Europe and North America, whereas the prevalence in Western Africa is very low. 1 However, this observation may result from an environmental influence on the manifestations of SLE or from inadequate health systems in Africa that fail to recognize the condition.

Infections seem to be able to work in both ways; they can modulate the immune system protecting against autoimmunity, but can also trigger the disease. An association between SLE and Epstein-Barr virus (EBV) infection has been described in children?and this virus may trigger a flare due to 
antigenic mimicking (EBV protein EBNA-1 can cross-react with the self-antigen Ro1). An association between cytomegalovirus (CMV) and SLE has also been suggested.

Oestrogens increase the risk of the disease and are a recognized trigger to flare, which probably contributes to the higher prevalence of SLE in women. Women treated with hormonal replacement therapy (but not with oral contraceptives) have a higher risk of mild-to-moderate flare, $\underline{\underline{ }}$ and associations between SLE and early menarche, menstrual irregularities and early or surgical menopause have been described. Other environmental triggers reported include ultraviolet light, cigarette smoking and silica. Drugs implicated in drug-induced lupus include hydralazine, D-penicillamine, minocycline, lithium and more recently TNFa blockers.

\section{Pathologic mechanisms}

The complex pathogenesis of SLE seems to involve almost every component of the immune system that culminates in antibody formation. The principal mechanisms are listed here. $\underline{5}$

$\boldsymbol{B}$ - and $\boldsymbol{T}$-cell signalling abnormalities that lead to hyperactivity and hyper-responsiveness of these cells. The signalling abnormalities include an abnormal T-cell receptor (TCR) complex, alterations on proteins that influence T-cell response to inflammation in various ways (such as mitogen-activated protein kinase; MAPK), decreased concentrations of blunting molecules such as Lyn (LCK/Yes-related novel tyrosine kinase), impaired signalling via B-cell inhibitory receptor FcgRIIB, and a faster response to a Bcell proliferation stimulus such as APRIL or BLyS.

-An important part is played by T cells. Auto-antigen-specific T-cells have been described. T-cells stimulate B-cell proliferation and are necessary to the secretion of high-affinity class-switched IgG antibodies, in a process called T-lymphocyte help. These antibodies are strongly associated to tissue damage in SLE. $\underline{T}$ T regulatory cells, which suppress T helper cells and B cells, are impaired in SLE. $\underline{7}$ -Dysregulated apoptosis and defective clearance of cellular debris increasing auto-antigen exposure and tolerance breakdown. In SLE, apoptosis (particularly of T lymphocytes) is dysregulated in a Fas/Fas ligand-dependent pathway that is hyperexpressed and correlates with SLE activity and autoantibody concentrations. Abnormalities in the innate immune system, including phagocytes and complement, are also linked to impaired recognition and clearance of apoptotic bodies. Subsequently, abnormal prolonged exposure of nuclear antigens that undergo multiple alterations creates neoepitopes or uncovers hidden epitopes. The remaining apoptotic bodies then go through a process called secondary necrosis that leads to the release of even more nuclear material.

NET (neutrophil extracellular traps), which are a mechanism of defence against microorganisms, also play an important role in perpetuating the inflammation and exposure of dsDNA because they are not promptly degraded in SLE. The lower degradation of NET in SLE correlates with disease activity.

-Antibody formation with pathogenic potential. The presence of hyperactive and hyper-responsive Band T-cells and the prolonged exposure to nuclear antigens leads to the formation of auto-antibodies directed against nuclear structures that are the immunologic hallmark of SLE. 
Auto-antibodies have been reported to be found up to 10 years before the onset of symptoms in $85 \%$ of SLE patients. (pg 8) Deposition of auto-antibodies and complement with inflammation is identified in biopsies of various tissues of patients with SLE. The most widely studied antibody is anti-double stranded DNA (anti-dsDNA), the serum concentration of which correlates with disease activity. Other antibodies have been identified in biopsies and serum from patients with SLE, and the presence of each of these correlates with specific clinical manifestations (Table 2).

However, the pathogenic role of the auto-antibodies in SLE is not clear. Although these antibodies are found in kidney biopsies, where they bind directly with renal cells or to circulating nuclear components, which are then deposited in the renal glomerular basement membrane (leading to inflammation), the hypothesis of secondary binding to an already inflamed tissue is not excluded. In this case, the presence of auto-antibodies would be a marker of inflammation and not a cause of it. Another suggestion is that dsDNA antibodies are actually binding to nucleosomes; in other words, dsDNA is linked to histones and the histones (which are positively charged) are responsible for binding to negatively charged regions of renal tissue - the so-called 'histone bridge theory'.

Auto-antibodies lead to the formation of immune complexes that directly induce B cells to produce more auto-antibodies and enhance the toll-like receptor-IFN1 pathway that also stimulates B cells to differentiate into plasmablasts.

It is important to bear in mind that different auto-antibodies have different specificities and sensitivities. Table 1 shows the auto-antibodies which may be present in SLE. ANA has $95 \%$ sensitive but lacks specificity. This is present up to $20 \%$ of the normal healthy population, as well as in other types of autoimmune disease, malignancies, infections and medication use. (Wandstrat et al) A higher ANA titre, especially 1:640 and above would be considered significant and may suggest the presence of an autoimmune condition. A homogenous pattern of ANA is seen in patients with SLE, and linked to antiDNA and anti-nucleosome antibodies whereas a speckled pattern is linked to anti-ENA. Anti-Sm and antidsDNA both are rare in other rheumatic diseases and are highly specific but have low and moderate sensitivity, respectively. (Page 9) antibodies which are 3 times as common in black compared to white patients are associated with a higher mortality and morbidity. Anti-dsDNA enzyme-linked immunoassays (ELISA) is used in clinical practice to monitor lupus activity, unlike anti-Sm, the levels of which do not correlate with disease activity. DNA Crithidia is another test which may be used in the diagnostic process being more specific than the ELISA method.

\section{Classification criteria}

SLE can affect virtually any organ and system in the body. Classification criteria are a fundamental tool to ensure that a consistent definition is used in research as well as epidemiologic studies.

The American College of Rheumatology (Jaarsma, Beattie et al.) classification criteria, first published in 1971, and subsequently revised in 1982 and in 1997, have been the most widely used. In 
2012, the Systemic Lupus International Collaborating Clinics (SLICC) group published validated criteria that have higher sensitivity than the revised ACR criteria, and lower specificity (though not statistically significant), which might lead to the inclusion of more patients in clinical trials. Furthermore, the SLICC criteria showed some changes which may improve their use in clinical practice. Some criteria were reorganized in order to minimize overlaps (such as photosensitivity and malar rash) while others were redefined, such as neurological manifestations and arthritis. Among the immunological criteria, anti-nuclear antibodies (ANA), anti-dsDNA, anti-Sm and anti-phospholipid antibodies are now considered different criteria so that each can contribute to the classification of SLE. Low complement is a new criterion. Using the SLICC classification, at least four criteria from a list of clinical and immunological features (Table 2), including at least one clinical criterion and one immunological criterion, should be present in order for a patient to be deemed to have SLE. However, biopsy-proven lupus nephritis together with ANA and antidsDNA may also be sufficient. The mandatory presence of clinical and immunological features are useful to avoid including patients without clinical manifestations or without positive antibodies. ${ }^{3}$

\section{Management \\ Clinical features}

SLE has an unpredictable course. It progresses in acute flares and periods of remission, although there are probably long periods of inflammatory sub-clinical activity. It can affect virtually every organ and system in the body, and during the flares usually more than one organ is affected. (Table 3 ) In mild forms the joints and skin are the main organs affected. In moderate forms other organs are involved but it is severe disease, notably of the kidneys and heart, that may be life threatening. The individual patient tends to have the same pattern of presentation and affected organs during the course of the disease. However, this is not always the case.

Renal involvement occurs in $30-50 \%$ of patients with SLE. This usually occurs early on in the disease course. It has been shown that anti-dsDNA, anti-Sm, complement and anti-C1q antibodies are strongly associated with renal involvement. It is imperative that blood pressure and urine analysis are performed as part of the clinical assessment of lupus patients. Renal biopsy is performed to identify the presence of lupus nephritis (LN), with six stages of lupus nephritis (I - minimal mesangial proliferative LN , II - mesangial proliferative LN, III - focal LN, IV - diffuse LN, V - membranous LN, VI - advanced sclerosis). It is also important to be aware of other potential causes of renal impairment in SLE patients as a result of concurrent diseases such as diabetes mellitus or nephrotoxic drugs.

Neuropsychiatric SLE (NPSLE) is highly diverse, comprising of central and peripheral nervous system manifestations as well as focal to more diffuse syndromes. This results in diagnostic difficulty, particularly because this may be present without derangements in the typical markers of active lupus such as anti-dsDNA and complement. Disruption in blood brain barrier has been thought to be an important factor for the development of NPSLE. Headaches are a common neurological complaint in SLE 
patients, and although studies have suggested that this is not more common in SLE but not specific or linked to active disease. However, it is important to assess for the presence of red flag signs suggesting a subarachnoid haemorrhage, venous sinus thrombosis and meningitis. Reduction in cognitive function, 'brain fog' as well as depression are also other common complaints in patients with SLE.

Gastrointestinal symptoms are common in SLE, and are usually mild and related to infective causes or as a side effect of treatment of SLE. Gastrointestinal manifestations of SLE, which may be due to gut vasculitis presents challenging diagnostic problems and may result in increased morbidity if not identified and treated. Lupus enteritis is a potentially fatal GI manifestation of SLE, with symptoms varying from that of an acute abdomen, pseudo-obstruction or protein-losing enteropathy. (pg 60) Associations between SLE and inflammatory bowel disease have been reported in the literature, and IBD may present before or after the diagnosis of SLE. The prevalence of ulcerative colitis has been reported in SLE as 0.4$0.74 \%$, with Crohn's disease reported in less than $0.4 \%$. There also have been case reports where antiTNF blocking biologic agents were used in IBD patients, who subsequently developed ANA/anti-dsDNA and clinical manifestations of SLE.

Primary biliary cirrhosis, another autoimmune disease, has been reported to occur between 0.3 and $2.4 \%$ of patients with SLE. Autoimmune hepatitis has been reported to occur in $3-10 \%$ of patients with SLE 3-10\% with a higher incidence in patients juvenile SLE, coeliac disease in $0.15-2.4 \%$ of patients with SLE and PSC may also occur in SLE but this has been reported to be rare. Other autoimmune diseases may also co-exist with SLE, such as secondary Sjogren's syndrome, overlap syndromes with features of rheumatoid arthritis and scleroderma, hypothyroidism and anti-phospholipid syndrome.

The prognosis of SLE has changed markedly in the last 50 years. The 5-year survival rate has improved from $50 \%$ in the 1950 s to over $90 \%$ today. 1 Improvement in prognosis is mainly due to the advances in immunosuppressive therapy, dialysis and transplantation and in general management, including anti-hypertensive drugs and statins. In addition, the recognition and management of various comorbidities, such as osteoporosis and cardiovascular disease, have improved. However, the mortality rate is still increased, with a recent meta-analysis reporting the all-cause mortality to be 2.6 times that of the general population. In the last 40 years it has been recognized that cardiovascular disease is a major cause of death in SLE. Urowitz et al. described this phenomenon in 1976, reporting a bimodal mortality in SLE. In the first phase disease activity is the main cause of death, while later on in the course of the disease the main cause of death is cardiovascular disease. $\underline{8}$ and $\underline{9}$

Classical cardiovascular (CV) risk factors contribute to this increased risk, notably smoking $(20 \%$ of patients in most cohorts continue to smoke), hypertension and dyslipidemia. In addition, the sustained inflammation in autoimmune diseases leads to accelerated atherosclerosis. Thromboembolic events are also increased in SLE.

Patients with SLE have been found to have an increased risk of certain malignancies. In a large multi-centre international study by Bernatsky et al, SLE patients were found to be 3 times more likely to 
develop haematological malignancies, in particular, non-Hodgkin's lymphoma and leukaemia, and there is also a small increased risk in lung, thyroid and vulval cancers. The pre-cancerous stage, cervical dysplasia has been reported to have a higher incidence in patients with SLE, especially in patients who have been treated with cyclophosphamide. This highlights the importance of SLE patients adhering to national cervical screening programmes. Interestingly, there is a lower risk of developing breast, endometrial and ovarian cancers in women and prostate cancers in men. Pregnancy morbidity is higher in patients with SLE, which include miscarriages, intrauterine growth restriction and pre-term birth, with the presence of lupus nephritis and anti-phospholipid syndrome increasing this risk further. The risk of neonatal heart block is estimated to be $2 \%$ with anti-Ro antibody positive mothers, increasing further to $15 \%$ in the next pregnancy if neonatal heart block was present in the previous pregnancy. Well-controlled lupus for at least 6 months prior to conception optimizes pregnancy outcomes.

The susceptibility to infections has been thought to be due to an impaired immune response, with the most common infection being respiratory tract infections. This is further compounded by increased lupus activity, hypocomplementaemia, leucopaenia, steroid use with doses above $7.5 \mathrm{mg}$, immunosuppressants such as cyclophosphamide. The mortality rate of patients with SLE due to infections has been reported to be early 5 times that of the general population. Hydroxychloroquine has been suggested to have a protective effect against infection. In view of this, it is important to review the vaccination status in patients with SLE. Patients with SLE on immunosuppressive drugs may have a lower immune response to vaccinations and there is limited evidence surrounding vaccinations in SLE patients. However, non-live vaccinations have been thought to be safe in SLE patients on immunosuppressive drugs and it is advised that patients with SLE receive the appropriate immunisations especially against pneumococcus (Pneumovax) and the yearly influenza vaccine as well as for certain age groups, immunisations as per national vaccination schedule such as the human papilloma virus and meningococcus ACWY vaccination.

\section{Indices}

Since the 1980s, new tools have been created to capture disease activity (as a global or individual organ/system score), damage and quality of life in patients with SLE. Standard measures of these aspects are critical when comparing different groups of patients and when assessing the response to drugs.

The correlation between indices measuring different aspects of the disease is poor, suggesting that they are complementary and should all be measured.

The two most popular indices capturing disease activity are the British Isles Lupus Assessment Group (BILAG) index and the Systemic Lupus Erythematosus Disease Activity Index (SLEDAI). The BILAG index measures the activity in each organ system in five categories (A, B, C, D and E). Each item is classified by the clinician as not present, improving, the same, worse or new, based upon the principle of the 'physician's intention to treat'. 10 The BILAG index is the only tool that measures activity in 
each specific organ or system. Other indices specific for just one organ have been validated (e.g. the Cutaneous Lupus Erythematosus Disease Activity and Severity Index; CLASI). The other indices are global and provide a final score for the whole body's disease activity.

SLEDAl is the most commonly used of the global scores indices. It measures activity in the last 10 days (though it has been validated for the last 30 days). It is simpler than BILAG but has some flaws; it does not distinguish partial improvement or worsening of symptoms, and it is less sensitive to change than other instruments. 10 Complementary tools have been created to accomplish some of this, such as the SLE disease activity index 2000 responder index-50 (S2K RI-50) used to demonstrate incomplete but clinically significant $(\geq 50 \%)$ improvement in disease activity. The Safety of Estrogens in Lupus Erythematosus National Assessment (SELENA) Flair Index and the SLEDAI Flare Index are versions of SELENA and SLEDAI, respectively, which allow the discrimination between different grades of flares in different organ systems, based on the treatment decision.

SLEDAI and BILAG correlate well over time, though it seems that BILAG-2004 captures disease activity requiring treatment better than SLEDAI-2000. SELENA/SLEDAI and BILAG-2004 also agree in the identification of 'no flare' and 'severe flare' but they define flare in different ways. A standard definition of flare is essential for the purpose of research and because flares are predictors of disease outcome. 11 Accordingly, a committee of lupus specialists has reached consensus in defining flare as a 'measurable increase in disease activity in one or more organ systems involving new or worse clinical signs and symptoms and/or laboratory measurements. It must be considered clinically significant by the assessor and usually there would be at least consideration of a change or an increase in treatment'. .11 This definition emphasizes that the increase in disease activity should be measurable and should lead to the consideration of changing the treatment. Nevertheless, it may still be difficult to distinguish mild flares from some non-lupus symptoms and progressive organ damage with this definition.

Other global indices have been validated, namely ECLAM (European Consensus Lupus Activity Measurement), LAI (Lupus Activity Index) and SLAM (Systemic Lupus Activity Measurement, and its revised version SLAM-R); it is worth mentioning that the inclusion of patients' subjective symptoms in SLAM make it very sensitive to changes important to patients though it may be less accurate. Damage is another essential feature to measure. SLICC/ACR - Systemic Lupus International Collaborating Clinics/American College of Rheumatology damage index (SDI) was designed to capture permanent change after the diagnosis of SLE regardless of the cause, secondary to the disease itself or secondary to treatment. The manifestation must be irreversible or should be present for 6 months. This tool has important prognostic properties. Rahman et al. showed, importantly, that early damage measured by this index is associated with greater mortality in the first decade of follow-up. $\underline{12}$

The patient's perception of SLE is not captured by activity or damage indices. There are now several tools to capture this perception. Examples of generic tools that measure physical, psychological and social impact of the disease are Short Form-36 (SF-36), the most widely used, Euro Quality of Life scale (EQ-5D) and 20-Item Short Form Health Survey (SF-20). Generic tools allow comparison with other 
diseases and healthy populations, but the specific tools are more sensitive and specific to features that are particular important for patients with SLE; examples of specific tools are LupusQoL, the Systemic Lupus Erythematosus-Specific Quality of Life Questionnaire (SLEQOL) and the Systemic Lupus Erythematosus Quality of Life Questionnaire (L-QoL).

For clinical trials on belimumab a new index was used, the SLE responder index (SRI) that used SELENA-SLEDAI to determine global improvement, BILAG to evaluate each organ and system and physician's global assessment to guarantee that there was not a worsening in patient's overall condition.

\section{Treatment}

The treatment of SLE depends on the organ and systems involved and the severity of the disease, and can vary from topical medicines for skin disease or NSAIDs for musculoskeletal disease to aggressive systemic immunosuppression. As already reported, the prognosis of SLE has greatly improved, probably due to new drugs that have been designed and tested, and also because of a better general management. It is now clear that associated diseases like osteoporosis and cardiovascular disease should be prevented and diagnosed and treated early.

Osteoporosis is strongly associated with SLE and the World Health Organization (WHO) suggests regular dual X-ray absorptiometry (DXA) for a correct diagnosis of osteoporosis, to determine the fracture risk and monitor the effect of treatment.

Patients with SLE should also be screened for the presence of CV risk factors and CV disease. .9 Modifiable risk-factors such as hypertension and hyperlipidaemia should be identified and concurrently managed. The importance of smoking cessation should be emphasized for reduction of cardiovascular risk and may improve cutaneous lupus. ACE-inhibitors should be considered not only for blood pressure control, but to reduce the level of proteinuria in patients with lupus nephritis and progression towards endstage renal disease as suggested by Hahn et al. Durran-Barragan et al suggested that ACEi use may delay renal manifestation of SLE and is associated with a reduced disease activity. (Durran-Barragan 2008)

Patients should be advised to reduce exposure to strong sunlight and to use adequate sun protection (SPF 50+) especially those with cutaneous manifestations.

\section{Corticosteroids}

Corticosteroids have been the 'core' treatment for SLE and other autoimmune diseases since they were first used in rheumatoid arthritis in 1949. Their mechanism of action consists in the modification of genomic and non-genomic pathways, the latest being activated in higher dosages (prednisolone $>30$ $\mathrm{mg} /$ day) leading to immunosuppressive and anti-inflammatory effects.

The dose and route of administration of these drugs varies according to the organ(s) affected and the severity of the disease. Their mode of use is mostly determined by the physician's experience; there are no guidelines describing the ideal regimen. In mild disease there is usually a response to prednisolone 5$15 \mathrm{mg} /$ day or equivalent, and a sparing agent or antimalarial can also be used. In life-threatening and 
organ-threatening disease, methylprednisolone is used as a pulse intravenous therapy. Some patients do not respond sufficiently to corticosteroid agents and in some manifestations of SLE a combination with an immunosuppressive drug is more effective.

European League Against Rheumatism (EULAR) has published guidelines on low-dose glucocorticoid therapy. They recommend close monitoring of the adverse effects, especially in high-dose, since most are dose-dependent.

\section{Hydroxychloroquine}

The immunomodulating properties of antimalarial drugs and their benefit in rheumatic disease have been known since World War II. In the last 20 years new evidence has shown that this drug is suitable for many manifestations of SLE: it prevents, and can be used to treat, articular and skin flares; it protects against ultraviolet light; it improves sicca symptoms; it is associated with milder disease; and it gives a more favourable cardiovascular profile in many different ways (reduces cholesterol, reduces the risk of diabetes, reduces the risk of development of carotid plaque and it has antithrombotic properties). In patients with renal disease it also facilitates the response to mycophenolate mofetil (MMF) $\underline{13}$ and ACR's 2012 guidelines suggest that it should be given to every lupus patient with nephritis. It was also shown that it prevents damage in the kidneys and central nervous system 14 and, probably as a result of all of this, it reduces mortality. ${ }^{15}$ Although hydroxychloroquine is generally a safe drug, prescribers have to be aware of risks of ocular toxicity, and initiate appropriate ophthalmology surveillance for this our practice is to seek a detailed ophthalmology review after 5 years on the drug [at a dose of $400 \mathrm{mg} /$ day].

Cardiotoxicity is a serious but rare adverse effect, with case reports describing hydroxychloroquineinduced cardiomyopathy.

\section{Immunosuppressives}

Other immunosuppressive drugs have been used in severe manifestations of SLE. Lupus nephritis is the most common life-threatening manifestation and has been the working field for the research into the effects of new drugs.

The National Institutes of Health (NIH) studies in the 1980s established that cyclophosphamide (CYC)-containing regimens were more effective than corticosteroids alone when treating lupus nephritis. Importantly, in 2002, the Euro-Lupus Nephritis trial demonstrated that a low-dose CYC regimen was as effective as the high-dose NIH regimens and had fewer (although not statistically significant) adverse effects; $; 6$ it is currently the preferred CYC regimen but, because CYC is very toxic even in low-dose regimens and because a significant percentage of patients are still resistant, new drugs have been tested. The Aspreva Lupus Management Study (ALMS) trial17 showed that MMF is a good option for the induction phase because it is as effective as CYC. It also has fewer adverse effects than CYC and is more effective than azathioprine (AZA). It is important to bear in mind that cyclophosphamide and MMF are contraindicated in pregnancy and breastfeeding, and therefore, AZA, though inferior to MMF, is a good option during pregnancy or if the preservation of fertility is a concern. $\underline{18}$ 
CYC has been used to treat myositis, and gastrointestinal and pulmonary manifestations. MMF is also effective in refractory haematological and dermatological manifestations. $\frac{19}{9}$ n the ALMS trial it was shown that CYC and MMF are similarly effective in controlling extra-renal disease (in a group of patients with renal lupus). .20 For neuropsychiatric lupus there is a lack of evidence concerning the best treatment option but corticosteroids alone or in combination with CYC or AZA are recommended; other drugs are used in refractory cases.

Both tacrolimus and ciclosporin, via inhibition of calcineurin, inhibit the production of cytokines and lymphocyte proliferation, especially T-helper cells. There is some evidence that tacrolimus is effective in the induction treatment for lupus nephritis. Ciclosporin may be used as a corticosteroid-sparing drug in patients with normal renal function.

\section{Biologic therapy}

As the pathogenesis of lupus is becoming better understood, the biologics have an increased role to play. The most logical and widely used option is B cell depletion achieved by direct B cell elimination or inhibition of $B$ cell survival agents.

Belimumab is a human monoclonal lgG1 that binds to BLyS (also known as BAFF, B-cell activating factor), an important B-cell stimulator protein. In March 2011, belimumab became the first drug in 50 years to be approved by the Food and Drug Administration (FDA) for the treatment of SLE, and this has also now been approved by the National Institute of Clinical Excellence (NICE). BLISS-76 and BLISS-52 are the two clinical trials that showed belimumab's efficacy in reducing disease activity and preventing flares. It was shown to be effective in mucocutaneous and musculoskeletal manifestations, and particularly effective in a subset of autoantibody-positive patients (ANA titre $\geq 1: 80$ and/or anti-dsDNA antibody concentration $\geq 30 \mathrm{IU} / \mathrm{ml}$ ) with a low C3. It has not yet been established whether it is effective in lupus nephritis and CNS manifestations.

Rituximab is a chimeric mouse/human monoclonal antibody against the CD20 antigen that is found on the surface of pre-B cells maturing to memory $B$ cells. The binding triggers apoptosis of peripheral $B$ cells without avoiding the regeneration from stem cells. This is licensed for non-Hodgkin's lymphoma, chronic lymphocytic leukaemia (CLL), rheumatoid arthritis and granulomatous polyangiitis. Currently, rituximab is being used off-license to treat SLE, funded by NHS England.

In many open-label studies, rituximab has shown good clinical results in SLE patients with different features, such as fatigue, skin, arthritis, serositis and renal disease. It is safe and well tolerated when used either alone or in combination or with cyclophosphamide.

Rituximab's corticosteroid-sparing effect is of great importance. After Ezeonyeji et al.21showed the benefit of early treatment with rituximab, Condon et al. have recently reported the result of the first 50 patients treated with the Rituxilup protocol, using rituximab and MMF but no oral corticosteroids, with very good efficacy. $\underline{22}$ 
Disappointingly, the two randomized controlled studies, EXPLORER and LUNAR, failed to show benefit from adding rituximab to the ongoing immunosuppressive treatment in extra-renal and renal lupus patients, respectively. Inadequate trial design almost certainly played a part, as patients with severe and refractory disease were excluded and a high dose of corticosteroids was maintained, obscuring the efficacy of rituximab and its corticosteroid sparing effect.

Ofatumumab is a fully humanized monoclonal antibody against CD20 antigen which is used to treat CLL and which has been shown to be efficacious in rheumatoid arthritis. This has been demonstrated in case studies to be effective in treating SLE and is a promising option for cases where rituximab is not tolerated. BELONG, a phase III trial investigating ocrelizumab, another anti-CD20 agent in class III/IV lupus nephritis was terminated early when a higher risk of serious infections was observed in the ocrelizumab group in combination with MMF.

Epratuzumab is a humanized IgG antibody against the CD22 antigen, an extracellular molecule that regulates $B$ cell activation and interaction with $T$ cells. although a phase II trial showed encouraging results the phase III trial did not meet its endpoints.

Atacicept (which blocks two B-cell activating factors - BLyS and APRIL), has shown promising results in both flare-prevention and active disease trials. In the APRIL-SLE phase II/III trial, atacicept $150 \mathrm{mg}$ was shown to be associated with a reduced flare-rate. Unfortunately, two fatal pulmonary infections led to the appropriate termination of the atacicept group in the trial [since deaths in small numbers of SLE patients have been reported in every SLE trial]. Subsequently, the ADDRESS II comparing atacicept $75 \mathrm{mg}$ and $150 \mathrm{mg}$ with placebo in treating moderately active SLE has shown atacicept to be efficacious at reducing disease activity as well as in flare-prevention with no increase in the risk of serious adverse events.

Tabalumab, is a humanised IgG4 antibody which targets BLyS, investigated in phase III trials, ILLUMINATE I and II. Although the primary end point was met in ILLUMINATE II, the results were not considered significant enough to continue further work.

Anifrolumab, rontalizumab and sifalizumab are Interferon alpha blocking agents. Phase II trial with rontalizumab did not meet its primary outcome. Sifalizumab and anifrolumab have shown promising results in preliminary trials, especially in those with a high interferon $\alpha$ signature.

A variety of other monoclonal antibodies including TNFa blockers, IL-6 receptor blocker tocilizumab and abatacept (which blocks the links between antigen-presenting cells and T cells) have also been used in relatively small numbers of patients with variable benefit. Eculizumab, a fully humanized IgG2/lgG4 monoclonal antibody against C5 has also undergone phase I trial. no drug apart from Benlysta has been approved for the treatment of SLE and further data are needed to support their use.

\section{Remission}

Treat to target for SLE (T2T/SLE), an international initiative was established to provide recommendations to treat according to a target identified for each patient -). 'Remission of systemic symptoms and organ 
manifestations' was named to be one of the targets. Treatment of SLE can be aimed to treat active disease, towards a lupus low disease activity state (LLDAS) to remission with treatment, with the ultimate aim of remission off treatment. (Morand 2016) Remission in SLE is currently a controversial topic. Studies on remission in SLE have used various definitions of remission including absence of clinical and serological activity, serological activity but clinically quiescent, and whether these states are achieved on or off treatment, A UK study reported complete disease remission of at least 3 years in $14.5 \%$ (MedinaQuinones, 2016 \#368) whilst another study reported prolonged disease remission of at least 5 years in $37 \%$. (Zen, 2015 \#366) A study by Wilhelm et al, however, reported durable disease remission to be a rare entity in SLE. They reported medians of 1.8 and 3.1 years respectively to achieve clinical and complete remission on treatment and 8.7 and 11.0 years respectively to achieve clinical and complete remission. In this study, patients of African-American origin and baseline C3 and C4 hypocomplementaemia were found to be associated with a longer period of time to remission. (Wilhelm, 2017 \#370)

Due to the lack of a consensus on the definition of remission, a large international task force on definitions of remission in SLE (DORIS) published in 2016 eight key statements and three principles for defining remission. The three principles stated are 1. That the definition of remission would be worded as 'Remission is a durable state characterized by ... (symptoms, signs, tests) 2 . A validated index is to be used to define remission, such as the BILAG 2004 score D or E, SLEDAI = 0, ECLAM = 0, complimented by a physician global assessment below a defined threshold 3 . The presence or absence of treatment has to be distinguished when defining remission. (van Vollenhoven, Voskuyl et al. 2017) A Dutch cohort study suggested that improved outcomes and reduced damage accrual were seen in patients with prolonged remission and LLDAS (Tsang, Bultink et al. 2017), with a study of 293 Caucasian patients showing that two years is the minimum number of years of disease remission to be associated with reduced damage accrual (Zen, laccarino et al. 2015). 


\section{Key points:}

1. SLE is a complex disease with multi-systemic involvement.

2. SLE is more common in females.

3. There is a higher prevalence of SLE in patients with African heritage, with more severe disease and poorer clinical outcomes.

4. Classification criteria and tools to measure disease activity should be used in clinical practice.

5. The SLICC classification criteria (2012) are more sensitive than the revised ACR criteria; requiring at least one clinical and one immunological feature to be present.

6. Biologics are effective for treating different features of SLE and have an important corticosteroidsparing effect.

\section{Self-assessment questions}

1. A 30-year-old lady with a history of SLE with previous lupus nephritis and extra-renal flares requiring cyclophosphamide, visits your clinic to discuss her plans regarding starting a family. Her disease has been stable for the last six months on $400 \mathrm{mg}$ of hydroxychloroquine and $750 \mathrm{mg}$ twice daily of mycophenolate mofetil. What advice would you give regarding her current medication regime?

a) Advice no change to her current medication MMF is contraindicated in pregnancy

b) Stop hydroxychloroquine and MMF Hydroxychloroquine is safe in pregnancy

c) Continue hydroxychloroquine and replace MMF with prednisolone Hydroxychloroquine is safe in pregnancy, and although prednisolone is also safe in pregnancy, this should be considered during disease flares, rather than for long term use

d) Continue hydroxychloroquine and replace MMF with azathioprine Hydroxychloroquine and azathioprine are safe in pregnancy. As SLE can flare during pregnancy and given her history of lupus nephritis requiring intravenous immunosuppressive therapy, this combination therapy would reduce her chances of developing a flare during pregnancy,

e) Continue hydroxychloroquine monotherapy Hydroxychloroquine is safe in pregnancy. SLE can flare during pregnancy and given her history, hydroxychloroquine alone may not be sufficient to control her disease during pregnancy.

2. A 40-year-old patient of African descent attends your clinic for a routine follow-up. She was diagnosed with SLE a year ago with a rash and arthralgia which has somewhat improved with 
hydroxychloroquine, but her fatigue persists. She also has a background of type II diabetes mellitus on metformin and hypertension for which she is on amlodipine. Her blood pressure is 145/98 and urine dip showed 3+ protein and a trace of blood. Urea $6.5 \mathrm{mmol} / \mathrm{L}$, creatinine 100 umol/L. What investigation would be most appropriate in identifying the cause of the abnormal urine dip in this patient?

a) Renal ultrasound

Renal ultrasound would be useful to assess for kidney size and hydronephrosis, but would not give a definite diagnosis of the urine dip abnormality.

b) Blood test for anti-dsDNA and complement levels

This may provide information about the patient's lupus activity but this does not provide a definitive diagnosis.

c) Renal biopsy

\section{A renal biopsy will be necessary to identify the aetiology of proteinuria.}

d) Urine protein:creatinine ratio and microscopy for casts

This is important as part of the work up in this lady, but this will not provide information regarding the aetiology of the proteinuria.

e) CT kidney/ureter/bladder

A CT scan is used to identify for causes of obstructive uropathy such as renal calculi but in this patient's case, will not be useful to identify the aetiology of her renal dysfunction.

3. A 50-year-old gentleman with psoriatic arthritis comes for a review in the rheumatology outpatients. He is currently treated with a TNF blocking drug, etanercept for the last six months after having failed combination disease-modifying therapy with methotrexate and sulfasalazine. He reports that he is unsure that his medication is controlling his disease as he has started to develop arthralgia. He has reported fatigue lately, with mouth ulcers and a photosensitive facial rash. His ANA, previously negative, was repeated in your clinic which shows 1:640 and otherwise has a normal auto-antibody screen. He has normal complement levels. What is the most likely diagnosis:

a) Treatment failure to etanercept Although this is possible, the additional symptoms in conjunction with a new positive ANA suggests drug-induced lupus

b) Allergic reaction to etanercept This is unlikely given the time interval between starting therapy and development of symptoms

c) Drug-induced lupus

This is now increasingly recognized in patients treated with anti-TNF therapy.

d) Systemic lupus erythematosus 
Although this is possible, the temporal association with the addition of a possible culprit drug suggests drug-induced lupus to be more likely

e) Severe erosive psoriatic arthritis

There is no suggestion in this story that this is the case. With the additional symptoms in conjunction with a new positive ANA, this suggests drug-induced lupus 


\begin{tabular}{|c|c|c|c|c|c|}
\hline Autoantibodies & $\begin{array}{l}\text { Prevalence } \\
\text { in SLE (\%) }\end{array}$ & $\begin{array}{l}\text { Sensitivity } \\
\text { in SLE (\%) }\end{array}$ & $\begin{array}{l}\text { Specificity in } \\
\text { SLE (\%) }\end{array}$ & $\begin{array}{l}\text { Clinical } \\
\text { manifestations }\end{array}$ & Other diseases \\
\hline $\begin{array}{l}\text { Anti-nuclear } \\
\text { antibody }\end{array}$ & $>95$ & High & Low & & $\begin{array}{l}\text { Scleroderma, Sjogren's syndrome, } \\
\text { rheumatoid arthritis, polymyositis, } \\
\text { dermatomyositis, drug-induced lupus, } \\
\text { infection, malignancy, drugs, other } \\
\text { autoimmune diseases }\end{array}$ \\
\hline Anti-dsDNA & $70-80$ & Moderate & High & Renal, skin & Rare in other diseases \\
\hline Nucleosomes & $60-90$ & High & High & Renal, skin & $\begin{array}{l}\text { Mixed connective tissue disease, } \\
\text { autoimmune hepatitis, scleroderma, } \\
\text { Sjogren's syndrome }\end{array}$ \\
\hline Anti-Ro/SSA & $30-40$ & Moderate & Moderate & $\begin{array}{l}\text { Sicca symptoms, } \\
\text { renal, skin, foetal } \\
\text { heart } \\
\text { abnormalities, } \\
\text { rash in newborn }\end{array}$ & $\begin{array}{l}\text { Sjogren's syndrome, rheumatoid } \\
\text { arthritis, polymyositis, scleroderma }\end{array}$ \\
\hline Anti-La/SSB & $15-20$ & Moderate & Moderate & $\begin{array}{l}\text { Foetal heart } \\
\text { abnormalities }\end{array}$ & Sjogren's syndrome \\
\hline Anti-Sm & $10-30$ & Low & High & $\begin{array}{l}\text { Renal, } \\
\text { neuropsychiatric, } \\
\text { associations with } \\
\text { higher mortality }\end{array}$ & Rare in other diseases \\
\hline Anti-U1-RNP & 25 & & & $\begin{array}{l}\text { Raynaud's } \\
\text { phenomenon, } \\
\text { lung, } \\
\text { neuropsychiatric, } \\
\text { musculoskeletal }\end{array}$ & $\begin{array}{l}\text { Mixed connective tissue disease, } \\
\text { scleroderma, polymyositis, } \\
\text { rheumatoid arthritis, Sjogren's } \\
\text { syndrome }\end{array}$ \\
\hline $\begin{array}{l}\text { Anti-Ribosomal } \\
\text { P }\end{array}$ & $10-40$ & Moderate & Moderate & $\begin{array}{l}\text { Neuropsychiatric, } \\
\text { hepatitis }\end{array}$ & \\
\hline Anti-NMDA & $33-50$ & & & Neuropsychiatric & Anti-NMDA receptor encephalitis \\
\hline $\begin{array}{l}\text { Anti- } \\
\text { Phospholipid }\end{array}$ & $20-40$ & Low & Low & $\begin{array}{l}\text { Thrombosis, } \\
\text { pregnancy loss }\end{array}$ & Malignancy, infection, drugs \\
\hline $\begin{array}{l}\text { Anti-Alpha- } \\
\text { actinin }\end{array}$ & 20 & & & Renal & \\
\hline Anti-C1q & $30-50$ & Low & Low & Renal & $\begin{array}{l}\text { Hypocomplementaemic urticarial } \\
\text { vasculitis, Sjogren's syndrome, } \\
\text { rheumatoid vasculitis }\end{array}$ \\
\hline Anti-Histone & $70-80$ & Low & Low & $\begin{array}{l}\text { Drug-induced } \\
\text { lupus }\end{array}$ & $\begin{array}{l}\text { Scleroderma, rheumatoid arthritis, } \\
\text { Sjogren's syndrome, mixed connective } \\
\text { tissue disease, vasculitis, malignancy, } \\
\text { liver disease }\end{array}$ \\
\hline
\end{tabular}

Table 1. Pathogenic auto-antibodies in SLE. Adapted from Rahman, A. \& Isenberg, D. A. Systemic lupus erythematosus, N Engl J Med358, 931 and Lloyd P, Doaty S, Hahn B, 
Aetiopathogenesis of systemic lupus erythematosus, Systemic Lupus Erythematosus, Oxford Rheumatology Library.

\section{SLICC classification system for SLE ${ }^{3}$}

\section{Clinical criteria \\ -Acute cutaneous lupus including:}

oLupus malar rash (do not count if malar discoid)

-Bullous lupus

oToxic epidermal variant of SLE

- Maculopapular lupus rash

oPhotosensitivity lupus rash

oOr subacute cutaneous lupus

-Chronic cutaneous lupus, including:

- Classic discoid rash, localized (above the neck) or generalized (above and below the neck)

oHypertrophic (verrucous) lupus

-Lupus panniculitis (profundus)

-Mucosal lupus

- Lupus erythematosus tumidus

-Chilblains lupus

-Oral ulcers

oDiscoid lupus/lichen planus overlap

-Palate - buccal or tongue

○Or nasal ulcers

-Nonscarring alopecia

-Synovitis involving two or more joints, characterized by swelling or effusion

Or tenderness in two or more joints and at least 30 min of morning stiffness.

-Serositis

oTypical pleurisy for more than 1 day

-Or pleural effusions

- Or pleural rub

-Typical pericardial pain

oOr pericardial effusion

-Or pericardial rub

-Renal

-Or pericarditis by electrocardiography

-Urine protein-to-creatinine ratio (or 24-h urinary protein) representing $500 \mathrm{mg}$

protein/24 $\mathrm{h}$

-Neurologic

or red cell casts

○Seizures

oPsychosis

-Mononeuritis multiplex

oMyelitis

-Peripheral or cranial neuropathy

-Acute confusional state

-Haemolytic anaemia

-Leucopenia $\left(<4000 / \mathrm{mm}^{3}\right.$ at least once)

Or lymphopenia $\left(<1000 / \mathrm{mm}^{3}\right)$ at least once

-Thrombocytopenia $\left(<100,000 / \mathrm{mm}^{3}\right)$ at least once

Immunologic criteria

-ANA

-Anti-dsDNA antibody

-Anti-Sm 
-Antiphospholipid antibody positivity as determined by any of the following:

oPositive test result for lupus anticoagulant

oFalse-positive test result for rapid plasma regain

-Medium- or high-titre anticardiolipin antibody

oPositive test result for anti- $\beta 2$-glycoprotein I

\section{-Low complement}

-Direct Coombs' test in the absence of haemolytic anaemia

ANA, antinuclear antibodies; SLE, systemic lupus erythematosus.

Adapted from Petri, M. et al. Derivation and validation of the Systemic Lupus International Collaborating Clinics classification criteria for systemic lupus erythematosus. Arthritis Rheum64, 2677-2686 (2012).

\section{Table 2}




\begin{tabular}{|c|c|}
\hline Clinical feature & Prevalence (\%) \\
\hline \multicolumn{2}{|l|}{ Dermatological } \\
\hline Butterfly malar rash & 40 \\
\hline Erythematous maculopapular eruption & 3 \\
\hline Discoid lupus & 20 \\
\hline Relapsing nodular non-suppurative panniculitis & $<5$ \\
\hline Vasculitic skin lesions & 40 \\
\hline Livedo reticularis & 20 \\
\hline Purpuric lesions & 40 \\
\hline Alopecia & 70 \\
\hline Oral ulceration & $7-52$ \\
\hline \multicolumn{2}{|l|}{ Musculoskeletal } \\
\hline Arthralgia/arthritis & 90 \\
\hline Tenosynovitis & 20 \\
\hline Myalgia & 50 \\
\hline Myositis & 5 \\
\hline \multicolumn{2}{|l|}{ Cardiopulmonary } \\
\hline Myocarditis & 3-15 \\
\hline Conduction disturbance & 10 \\
\hline Sinus tachycardia & $6-100$ \\
\hline Valvular dysfunction & 54 \\
\hline Pleurisy & 35 \\
\hline Pleural effusion & 25 \\
\hline Pericarditis & 15 \\
\hline Interstitial alveolitis/pneumonitis & $1-12$ \\
\hline Interstitial fibrosis & $3-13$ \\
\hline Shrinking lung syndrome & $0.6-0.9$ \\
\hline Pulmonary hypertension & $0.5-43$ \\
\hline Coronary vasculitis & Rare \\
\hline \multicolumn{2}{|l|}{ Gastrointestinal } \\
\hline Abdominal pain & $8-40$ \\
\hline Nausea & 15 \\
\hline Vomiting & $<10$ \\
\hline Diarrhoea & $<10$ \\
\hline Intestinal vasculitis & $0.2-14$ \\
\hline Lupus peritonitis/Abdominal serositis & rare \\
\hline Lupus hepatitis & 9.3 \\
\hline Acute lupus cholecystitis & rare \\
\hline Lupus pancreatitis & $0.44-4$ \\
\hline \multicolumn{2}{|l|}{ Renal } \\
\hline Haematuria & 10 \\
\hline Proteinuria & 60 \\
\hline Casts & 30 \\
\hline \multicolumn{2}{|l|}{ Neuropsychiatric } \\
\hline \multicolumn{2}{|l|}{ Central Nervous System } \\
\hline Depression & 15 \\
\hline Acute confusional state & 3-7 \\
\hline Psychosis & $5-10$ \\
\hline Anxiety & $7-24$ \\
\hline Seizures & $7-20$ \\
\hline Hemiplegia & 10 \\
\hline Cranial nerve lesions & 10 \\
\hline Cerebellar ataxia & 5 \\
\hline Aseptic meningitis & $0.4-3$ \\
\hline Headache (Migraine, benign intracranial hypertension) & $21-57$ \\
\hline Demyelinating syndrome & $0.2-2$ \\
\hline Myelopathy & $1-3$ \\
\hline Cerebrovascular disease & $2-15$ \\
\hline Cognitive dysfunction & $27-80$ \\
\hline Chorea/Movement disorder & 1 \\
\hline Peripheral nervous system & \\
\hline Acute inflammatory demyelinating polyradiculopathy & $0.2-0.6$ \\
\hline Mononeuropathy & $1-7$ \\
\hline Polyneuropathy & $2.8-28$ \\
\hline Myasthenia gravis & $0.2-2$ \\
\hline Cranial neuropathy & $1.5-7$ \\
\hline Haematological & \\
\hline Anaemia & \\
\hline Iron deficiency & 30 \\
\hline Chronic disease & 75 \\
\hline Autoimmune haemolytic & $3-15$ \\
\hline Leucopaenia & $30-60$ \\
\hline Lymphopaenia & 76 \\
\hline Thrombocytopaenia & $15-25$ \\
\hline Ophthalmic & \\
\hline Orbital inflammation, myositis, proptosis & $\mathrm{n} / \mathrm{a}$ \\
\hline Keratoconjuncivitis sicca & 25 \\
\hline Keratitis & $\mathrm{n} / \mathrm{a}$ \\
\hline Episcleritis/scleritis & $\mathrm{n} / \mathrm{a}$ \\
\hline Anterior/posterior uveitis & $0.1-4.8$ \\
\hline Optic neuropathy: neuritis, ischaemic neuropathy, papilloedema & 1 \\
\hline Retinal disease & $3-29$ \\
\hline
\end{tabular}


Table 3. Prevalence of clinical features in SLE. 


\section{KEY REFERENCES}

1 Pons-Estel GJ, Alarcon GS, Scofield L, Reinlib L, Cooper GS. Under- standing the epidemiology and progression of systemic lupus erythematosus. Semin Arthritis Rheum 2010; 39: 257-68.

2 Alarcon GS, Calvo-Alén J, McGwin Jr G, et al. Systemic lupus erythematosus in a multiethnic cohort: LUMINA XXXV. Predictive factors of high disease activity over time. Ann Rheum Dis 2006; 65: 1168-74. 3 Petri M, Orbai AM, Alarcon GS, et al. Derivation and validation of the Systemic Lupus International Collaborating Clinics classification criteria for systemic lupus erythematosus. Arthritis Rheum 2012; 64: 2677-86.

4 Sullivan KE. Genetics of systemic lupus erythematosus: clinical implications. Rheum Dis Clin North Am 2000; 26: 229-56.

5 Gatto M, Zen M, Ghirardello A, et al. Emerging and critical issues in the pathogenesis of lupus. Autoimmun Rev 2013; 12: 523-36.

6 Flesher DLT, Sun X, Behrens TW, Graham RR, Criswell LA. Recent advances in the genetics of systemic lupus erythematosus. Expert Rev Clin Immunol 2010; 6: 461-79.

7 Rahman A, Isenberg DA. Systemic lupus erythematosus. N Engl J Med 2008; 358: 929-39.

8 Urowitz MB, Bookman AA, Koehler BE, Gordon DA, Smythe HA, Ogryzlo MA. The bimodal mortality pattern of systemic lupus erythematosus. Am J Med 1976; 60: 221-5.

9 Skaggs BJ, Hahn BH, McMahon M. Accelerated atherosclerosis in patients with SLE e mechanisms and management. Nat Rev Rheumatol 2012; 8: 214-23.

10 Romero-Diaz J, Isenberg D, Ramsey-Goldman R. Measures of adult systemic lupus erythematosus: updated version of British Isles Lupus Assessment Group (BILAG 2004), European Consensus Lupus Activity Measurements (ECLAM), Systemic Lupus Activity Measure, Revised (SLAM-R), Systemic Lupus Activity Questionnaire for Population Studies (SLAQ), Systemic Lupus Erythematosus Disease Activity Index 2000 (SLEDAI-2K), and Systemic Lupus International Collaborating Clinics/American College of Rheumatology Damage Index (SDI). Arthritis Care Res (Hoboken) 2011; 63(suppl 11): S37-46.

11 Ruperto N, Hanrahan LM, Alarcon GS, et al. International consensus for a definition of disease flare in lupus. Lupus 2011; 20: 453-62.

12 Rahman P, Gladman DD, Urowitz MB, Hallett D, Tam LS. Early damage as measured by the SLICC/ACR damage index is a predictor of mortality in systemic lupus erythematosus. Lupus $2001 ; 10$ : 93-6.

13 Kasitanon N, Fine DM, Haas M, Magder LS, Petri M. Hydroxychloroquine use predicts complete renal remission within 12 months among patients treated with mycophenolate mofetil therapy for membranous lupus nephritis. Lupus 2006; 15: 366-70.

14 Fessler BJ, Alarcon GS, McGwin Jr G, et al. Systemic lupus erythe- matosus in three ethnic groups: $\mathrm{XVI}$. Association of hydroxychloroquine use with reduced risk of damage accrual. Arthritis Rheum 2005; 52: $1473-80$.

15 Alarcon GS, McGwin G, Bertoli AM, et al. Effect of hydroxychloroquine on the survival of patients with systemic lupus erythematosus: data from LUMINA, a multiethnic US cohort (LUMINA L). Ann Rheum Dis 2007; 66: $1168 \mathrm{e} 72$.

16 Houssiau FA, Vasconcelos C, D'Cruz D, et al. Immunosuppressive therapy in lupus nephritis: the Euro-Lupus Nephritis Trial, a randomized trial of low-dose versus high-dose intravenous cyclophosphamide. Arthritis Rheum 2002; 46: 2121-31.

17 Appel GB, Contreras G, Dooley MA, et al. Mycophenolate mofetil versus cyclophosphamide for induction treatment of lupus nephritis. J Am Soc Nephrol 2009; 20: 1103-12. SYSTEMIC LUPUS ERYTHEMATOSUS MEDICINE 42:3 1322014 Published by Elsevier Ltd.

18 Dooley MA, Jayne D, Ginzler EM, et al. Mycophenolate versus azathioprine as maintenance therapy for lupus nephritis. N Engl J Med 2011; 365: 1886-95.

19 Mok CC. Mycophenolate mofetil for non-renal manifestations of systemic lupus erythematosus: a systematic review. Scand J Rheumatol 2007; 36: 329-37.

20 Ginzler EM, Wofsy D, Isenberg D, et al. Nonrenal disease activity following mycophenolate mofetil or intravenous cyclophosphamide as induction treatment for lupus nephritis: findings in a multicenter, prospective, randomized, open-label, parallel-group clinical trial. Arthritis Rheum 2010; 62: 211-21. 21 Ezeonyeji AN, Isenberg DA. Early treatment with rituximab in newly diagnosed systemic lupus erythematosus patients: a steroid-sparing regimen. Rheumatology 2012; 51: 476-81. 
22 Condon M, Ashby D, Pepper RJ, et al. Prospective observational single centre cohort study to evaluate the effectiveness of treating lupus nephritis with rituximab and mycophenolate mofetil but no oral steroids. Ann Rheum Dis 2013; 72: 1280e6.

23 Wallace D, Goldenberg D. Epratuzumab for systemic lupus erythematosus. Lupus 2013; 22: 400e5.

Cistaro, A., F. Paze, S. Durando, M. Cogoni, R. Faletti, S. Vesco, S. Vallero, N. Quartuccio, G. Treglia and U. Ramenghi (2014). "Autoimmune lymphoproliferative syndrome and non-Hodgkin lymphoma: what 18Ffluorodeoxyglucose positron emission tomography/computed tomography can do in the management of these patients? Suggestions from a case report." Rev Esp Med Nucl Imagen Mol 33(2): 99-102. Jaarsma, T., J. M. Beattie, M. Ryder, F. H. Rutten, T. McDonagh, P. Mohacsi, S. A. Murray, T. Grodzicki, I. Bergh, M. Metra, I. Ekman, C. Angermann, M. Leventhal, A. Pitsis, S. D. Anker, A. Gavazzi, P. Ponikowski, K. Dickstein, E. Delacretaz, L. Blue, F. Strasser, J. McMurray and H. F. A. o. t. E. S. C. Advanced Heart Failure Study Group of the (2009). "Palliative care in heart failure: a position statement from the palliative care workshop of the Heart Failure Association of the European Society of Cardiology." Eur J Heart Fail 11(5): 433-443.

Lewis, M. J. and A. S. Jawad (2016). "The effect of ethnicity and genetic ancestry on the epidemiology, clinical features and outcome of systemic lupus erythematosus." Rheumatology (Oxford). Morand, E. F. (2016). "Connective tissue diseases: Remission in SLE - are we there yet?" Nat Rev Rheumatol 12(12): 696-698.

Tsang, A. S. M. W., I. E. Bultink, M. Heslinga and A. E. Voskuyl (2017). "Both prolonged remission and Lupus Low Disease Activity State are associated with reduced damage accrual in systemic lupus erythematosus." Rheumatology (Oxford) 56(1): 121-128.

van Vollenhoven, R., A. Voskuyl, G. Bertsias, C. Aranow, M. Aringer, L. Arnaud, A. Askanase, P. Balazova, E. Bonfa, H. Bootsma, D. Boumpas, I. Bruce, R. Cervera, A. Clarke, C. Coney, N. Costedoat-Chalumeau, L. Czirjak, R. Derksen, A. Doria, T. Dorner, R. Fischer-Betz, R. Fritsch-Stork, C. Gordon, W. Graninger, N. Gyori, F. Houssiau, D. Isenberg, S. Jacobsen, D. Jayne, A. Kuhn, V. Le Guern, K. Lerstrom, R. Levy, F. Machado-Ribeiro, X. Mariette, J. Missaykeh, E. Morand, M. Mosca, M. Inanc, S. Navarra, I. Neumann, M. Olesinska, M. Petri, A. Rahman, O. P. Rekvig, J. Rovensky, Y. Shoenfeld, J. Smolen, A. Tincani, M. Urowitz, B. van Leeuw, C. Vasconcelos, A. Voss, V. P. Werth, H. Zakharova, A. Zoma, M. Schneider and M. Ward (2017). "A framework for remission in SLE: consensus findings from a large international task force on definitions of remission in SLE (DORIS)." Ann Rheum Dis 76(3): 554-561.

Zen, M., L. laccarino, M. Gatto, S. Bettio, L. Nalotto, A. Ghirardello, L. Punzi and A. Doria (2015). "Prolonged remission in Caucasian patients with SLE: prevalence and outcomes." Ann Rheum Dis 74(12): 2117-2122. 